\title{
Bilateral Carotid Dissection Presenting as Status Migrainosus: All That Glitters Is Not Gold
}

\author{
Aida Rodriguez Sainz ${ }^{\mathrm{a}}$, Itxaso Azkune Calle ${ }^{\mathrm{a}}$, Jose Maria Ontanon ${ }^{\mathrm{a}}$, \\ Marian Gomez Beldarrain ${ }^{\mathrm{a}}$, Juan Carlos Garcia-Monco ${ }^{\mathrm{a}, \mathrm{b}}$
}

\begin{abstract}
We present a patient who was admitted with status migrainosus caused by spontaneous bilateral carotid dissection. Cervical artery dissection commonly presents with headache and neck pain, usually of sudden onset or of different characteristic in patients with a history of primary headache. Occasionally, artery dissection mimics a migraine attack; yet, its presentation as a status migrainosus has not been reported so far.
\end{abstract}

Keywords: Carotid dissection; Status migrainosus; Artery dissection

\section{Introduction}

Status migrainosus is defined as a debilitating migraine attack lasting for more than $72 \mathrm{~h}$ and not attributed to another disorder [1].

Cervical artery dissection commonly presents with headache and neck pain, usually of sudden onset or of different characteristic in patients with a history of primary headache. The criteria for headache attributed to arterial dissection include any new headache, facial pain or neck pain of acute onset, with or without other neurological symptoms or signs, demonstrated by appropriate imaging investigations, in close temporal relation to and on the same side as the dissection, which resolves within 1 month [1]. Occasionally, artery dissection mimics a migraine attack; yet, its presentation as a status migrainosus has not been reported so far.

\footnotetext{
Manuscript accepted for publication May16, 2013

${ }^{a}$ Department of Neurology and Magnetic Resonance Unit, OSATEK, Hospital de Galdakao, Barrio Labeaga s/n, C.P. 48960 Galdakao,Vizcaya, Spain

${ }^{\mathrm{b}}$ Corresponding Author: Juan Carlos Garcia-Monco, Department of Neurology, Hospital de Galdakao, Vizcaya, Spain.

Email: hospit05@sarenet.es
}

doi: http://dx.doi.org/10.14740/jmc1306w
We here present a migraine patient who presented with status migrainosus caused by spontaneous bilateral carotid dissection.

\section{Case Report}

A 42-year-old woman consulted for a holocranial, throbbing and severe headache. She had had episodic migraines without aura since adolescence that she managed with non-steroidal anti-inflammatory drugs (NSAIs). On average, attacks lasted for 1 to 3 days and often coincided with her menses.

Otherwise, her past history was irrelevant. She had been taking low-dose contraceptives for several years with regular medical controls.

She consulted for persistent headache of gradual onset that she described as similar to prior attacks but of longer duration. The onset of pain again was catamenial. At the time of consultation pain had been present for 10 days and responded poorly to NSAIs or triptans. A cranial contrastenhanced CT scan had been normal. She denied any prior craniocervical trauma.

With a diagnosis of status migrainosus she was placed on oral steroids (tapering schedule), omeprazole, metoclopramide, amitriptyline and triptans for bouts of severe pain.

Over the following 7 days pain intensity lessened but was still severe. She was treated with intravenous valproic acid (push of $400 \mathrm{mg}$ ) followed by an oral schedule (500 mg twice a day).

Two weeks later she had improved significantly. She then referred an occasional, intermittent bruit on the left upper neck. A cranial MRI with angiography of the neck vessels was performed that disclosed a bilateral carotid dissection with occlusion on the right side and evidence of intramural thrombus on the left side (Fig. 1).

The patient was placed on coumadin for 3 months, and has remained well ever since.

\section{Discussion}

This patient presented with a headache that fulfilled the time 


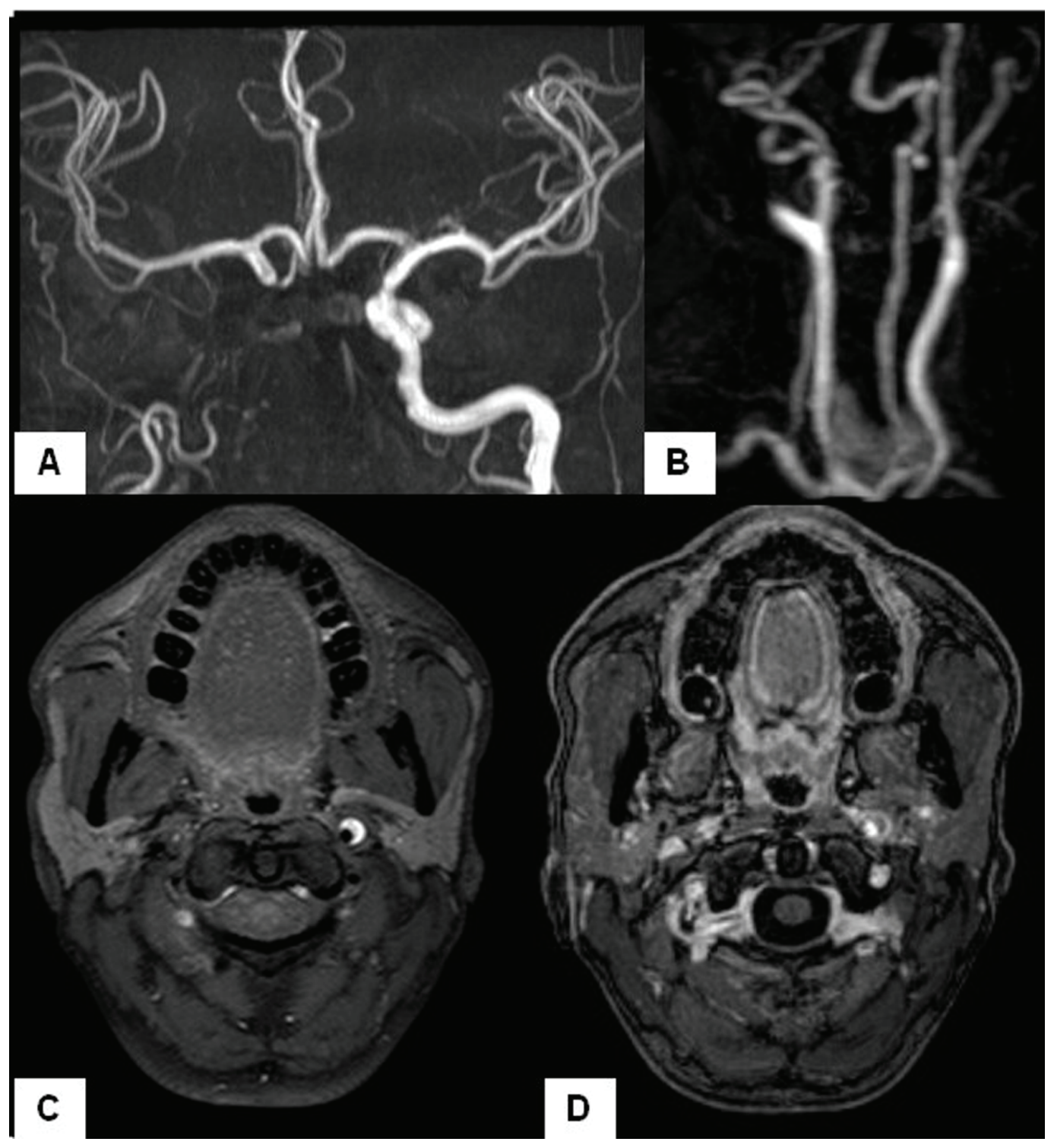

Figure 1. MR-angiography of intracranial $(A)$ and cervical vessels $(B)$ shows the absence of the ICA except for a short proximal segment of sharpened morphology. (C) Cranial MRI shows the typical half-moon hyperintense signal on the left ICA wall before its entrance into the petrous canal, consistent with dissection (T1-weighted, fat saturation sequences). Notice the absence of representation of the right carotid artery at the same level. (D) After gadolinium administration no decrease in the lumen of the left ICA was observed, also indicating arterial dissection.

criteria for status migrainosus: persistent headache with migraine features for $>72$ consecutive hours. Over the years, status migrainosus has represented an emergency in terms of management among patients with migraine [2]. This term, however, probably needs to be revised, since the availability of more effective migraine abortive therapy (triptans) in the last decade has resulted in a faster resolution of acute attacks and, hence, in less status migrainosus. Furthermore, at present the most common situation fulfilling the criteria for status migrainosus is chronic migraine with medication overuse [1].

Pain in cervical artery dissection lacks a specific pattern and is sometimes misleading, mimicking other headaches such as migraine, cluster headache, primary thunderclap headache and SAH (particularly since intracranial vertebral artery dissection can itself present with $\mathrm{SAH}$ ).

Arterial dissection needs to be excluded as a secondary mimic in several conditions, including migraine with aura, exertional headache, orgasmic headache and thunderclap headache [3]. In fact, headache is the most common complaint in patients with cervical artery dissection; yet, its presentation as a status migrainosus is quite unusual. In a series of 65 patients with ICA dissection with isolated headache, $74 \%$ complained cephalic pain that was homolateral to the 
dissection in $79 \%$ of cases, and was associated to signs of cerebral or retinal ischemia in $79 \%$ of patients [4].

Pain in cervical artery dissection lacks a specific pattern and usually other associated features, such as a painful Horner's syndrome or a painful tinnitus of sudden onset, providing the clue to the diagnosis. Our patient developed an intermittent left-sided, non-painful cervical bruit that led to perform an MR-angiography that revealed the bilateral carotid dissection.

Cervical arterial dissection causes referred pain that is associated with the nerves distributed to the vessels. Thus, while in vertebral artery dissection cervical and occipital pain is frequent, with dissection of the internal carotid artery (ICA), temporal and facial pain seems more frequent [5]. The fact that this patient had a bilateral dissection could have contributed to a diffuse, holocephalic headache rather than to unilateral pain.

In conclusion, bilateral carotid artery dissection needs to be excluded in patients with status migrainosus, a term that should be revised since at present is of little clinical usefulness.

\section{Disclosures}

None of the authors reports any disclosures.

\section{References}

1. The International Classification of Headache Disorders: 2nd edition. Cephalalgia. 2004;24(Suppl 1):9-160.

2. Friedman BW, Lipton RB. Headache emergencies: diagnosis and management. Neurol Clin. 2012;30(1):43-59, vii.

3. Arnold M, Cumurciuc R, Stapf C, Favrole P, Berthet $\mathrm{K}$, Bousser MG. Pain as the only symptom of cervical artery dissection. J Neurol Neurosurg Psychiatry. 2006;77(9):1021-1024.

4. Biousse V, Woimant F, Amarenco P, Touboul PJ, Bousser MG. Pain as the only manifestation of internal carotid artery dissection. Cephalalgia. 1992;12(5):314-317.

5. Mokri B. Headaches in cervical artery dissections. Curr Pain Headache Rep. 2002;6(3):209-216. 